\title{
Solving multi-choice multi-objective transportation problem: a utility function approach
}

\author{
Gurupada Maity and Sankar Kumar Roy*
}

\author{
*Correspondence: \\ sankroy2006@gmail.com \\ Department of Applied \\ Mathematics with Oceanology and \\ Computer Programming, \\ Vidyasagar University, Midnapore, \\ West Bengal 721102, India
}

\begin{abstract}
This paper explores the study of multi-choice multi-objective transportation problem (MCMTP) under the environment of utility function approach. MCMTP is converted to multi-objective transportation problems (MOTP) by transforming the multi-choice parameters like cost, demand, and supply to real-valued parameters. A general transformation procedure using binary variables is illustrated to reduce MCMTP into MOTP. Most of the MOTP are solved by goal programming (GP) approach. Using GP, the solution of MOTP may not be satisfied all the time by the decision maker (DM) when the proposed problem contains interval-valued aspiration level. To overcome this difficulty, here we propose the approaches of revised multi-choice goal programming (RMCGP) and utility function into the MOTP and then compared the solution between them. Finally, numerical examples are presented to show the feasibility and usefulness of our paper.
\end{abstract}

Keywords: Transportation problem; Multi-choice programming; Multi-objective decision making; Goal programming; Multi-choice programming; Utility function

\section{Introduction}

The transportation problem is the central nerve system to keep the balance in economical world from ancient day until today. In earlier days, transportation problem developed with the assumption that the supply, demand, and cost parameters are exactly known. But in real-life applications, all the parameters of the transportation problem are not generally defined precisely. Keeping this point of view, in this paper, we have incorporated with multi-choice multi-objective transportation problem (MCMTP) considering the parameters of transportation problem as multi-choice type.

Instead of single choice, if there may be several choices involved associated with the transportation parameters like cost, supply, or demand, then the decision maker is confused to select the proper choice for these parameters. In this circumstances, the study of transportation problem creates a new direction which is called multi-choice multiobjective transportation problem. Chang [1] proposed a multi-choice goal programming approach to solve the mathematical programming. Again in the subsequent year, Chang [2] proposed another multi-choice goal programming approach in revised form. Though the multi-choice concept discussed in both the papers of Chang [1,2] is totally

(c) 2014 Maity and Roy; licensee Springer. This is an Open Access article distributed under the terms of the Creative Commons Attribution License (http://creativecommons.org/licenses/by/2.0), which permits unrestricted use, distribution, and reproduction in any medium, provided the original work is properly credited. 
related to the goals of objective functions, recently, Mahapatra et al. [3] and Roy et al. [4] discussed the multi-choice stochastic transportation problem involving extreme value distribution and exponential distribution in which the multi-choice concept involved only in the cost parameters.

In this paper, we have designed a general transformation technique to reduce the multichoice parameters like cost, time, and demand to single-valued parameters. Using this technique, the MCMTP problem can be reduced to MOTP.

Goal programming (GP), an analytical approach, is devised to address the decisionmaking problem where targets have been assigned to all objective functions. The objective functions are conflicting and commensurable to each other, and the DM is interested to minimize the non-achievement of the corresponding goals. In other words, the DM derived an optimal solution with this strategy of GP which is satisfactory. However, using GP, the solution procedure for MOTP has some limitation. The main limitation behind GP is that the priority of goals for the DM is not easily considered. It seems far from reality. In the recent past, the notion of utility function is introduced by several researchers such as [5], Yu et al. [6], and Podinovski [7]. Recently, multi-choice goal programming (MCGP) has been proposed by Chang [1,2] to solve multi-objective decision-making problems with aspiration level. However, to the best of our knowledge, no works have been done on utility function to solve MOTP with the DM's preferences. The main motivation of this study is to investigate the better solution of MOTP by using utility function approach and then compare the solution to other methods such as GP and RMCGP.

Charnes et al. [8] introduced the concept of GP further developed by several researchers such as Charnes and Cooper [9], Lee [10], Ignizio [11], Tamiz et al. [12], and Romero [13]. In long back, the main concept of GP was to minimize the deviation between the achievement goals and the achievement levels. The mathematical model of multi-objective decision making (MODM) can be considered in the following form:

GP

$$
\min \sum_{i=1}^{K} w_{i}\left|Z^{i}(X)-g_{i}\right|
$$

subject to $x \in F(F$ is the feasible set)

where $w_{i}$ are the weights attached to the deviation of the achievement function. $Z^{i}(X)$ is the $i$ th objective function of the $i$ th goal, and $g_{i}$ is the aspiration level of the $i$ th goal. $\left|Z^{i}(X)-g_{i}\right|$ represents the deviation of the $i$ th goal. Later on, a modification on GP is provided and denoted as weighted goal programming (WGP) which can be displayed in the following form:

WGP

$$
\begin{array}{r}
\min \sum_{i=1}^{K} w_{i}\left(d_{i}^{+}+d_{i}^{-}\right) \\
\text {subject to } Z^{i}(X)-d_{i}^{+}+d_{i}^{-}=g_{i} \\
d_{i}^{+} \geq 0, d_{i}^{-} \geq 0, \quad i \in\{1,2, \ldots, K\} \\
x \in F(F \text { is the feasible set })
\end{array}
$$

where $d_{i}^{+}$and $d_{i}^{-}$are over and under achievements of the $i$ th goal, respectively. 
However, the conflicts of resources and the incompleteness of available information make it almost impossible for DMs to set the specific aspiration levels and choose the better decision. To overcome this situation, MCGP approach has been presented by Chang [1] with a new direction to solve MODM problem. In the next year, Chang [2] proposed the revised form of MCGP defined as RMCGP to solve MODM. The mathematical model of MODM using RMCGP is defined as follows:

\section{RMCGP}

$$
\min \sum_{i=1}^{K} w_{i}\left(d_{i}^{+}+d_{i}^{-}\right)+\alpha_{i}\left(e_{i}^{+}+e_{i}^{-}\right)
$$

subject to $Z^{i}(X)-d_{i}^{+}+d_{i}^{-}=y_{i}, \quad i=1,2, \ldots, K$

$$
\begin{array}{r}
y_{i}-e_{i}^{+}+e_{i}^{-}=g_{i, \max }, \text { or } g_{i, \min } \quad i=1,2, \ldots, K \\
g_{i, \min } \leq y_{i} \leq_{i, \max }, \quad i=1,2, \ldots, K \\
d_{i}^{+}, d_{i}^{-}, e_{i}^{+}, e_{i}^{-} \geq 0, \quad i=1,2, \ldots, K \\
x \in F(F \text { is the feasible set })
\end{array}
$$

where $y_{i}$ is the continuous variable associated with $i$ th goal which restricted between the upper $\left(g_{i, \max }\right)$ and lower $\left(g_{i, \min }\right)$ bounds, $e_{i}^{+}$and $e_{i}^{-}$are positive and negative deviations attached to the $i$ th goal of $\left|y_{i}-g_{i, \max }\right|$, and $\alpha_{i}$ is the weight attached to the sum of the deviations of $\left|y_{i}-g_{i, \max }\right|$; other variables are defined as in WGP.

The main motivation of this paper is to investigate the better solution of MCMTP by using utility function approach and then compare the solutions to other methods such as GP and RMCGP.

The remainder of the paper is organized as follows: In Section "Mathematical model", mathematical models are formulated for MOTP and MCMTP and solution procedures have been discussed with utility function approach. In the next section "Numerical examples", we demonstrate the usefulness of the proposed model with realistic examples. Finally, conclusion is presented regarding our consideration.

\section{Mathematical model}

The mathematical model of multi-objective transportation problem (MOTP) can be considered as follows:

\section{Model 1}

$$
\begin{aligned}
& \min : Z^{t}=\sum_{i=1}^{m} \sum_{j=1}^{n} C_{i j}^{t} x_{i j}, \quad t=1,2, \ldots, K \\
& \text { subject to } \sum_{j=1}^{n} x_{i j} \leq a_{i}, \quad i=1,2, \ldots, m \\
& \sum_{i=1}^{m} x_{i j} \geq b_{j}, j=1,2, \cdots, n \\
& \text { and } x_{i j} \geq 0, \quad \forall i \text { and } j
\end{aligned}
$$

Here $C_{i j}^{t}, a_{i}, b_{j}$ are the cost, supply, and demand parameters of $t$ th objective function in MOTP, respectively, and $\sum_{i=1}^{m} a_{i} \geq \sum_{j=1}^{n} b_{j}$ is the feasibility condition. According to the nature of the problem, the decision maker has the right to choose the goals of the objective 
functions. Assuming that these goals are $g_{t}(t=1,2, \ldots, K)$ of $K$ objective functions, the goals are defined as interval valued as $g_{t}=\left[g_{t, \min }, g_{t, \max }\right],(t=1,2, \ldots, K)$.

In many real-life situations, the multiple choices in the transportation parameters like cost, demand, and source create complexities for the DM to make a decision. Multi-choice costs may occur due to several routes for transporting the goods. Due to weather condition or different seasons, the demands or the supply become multi-choices in nature. In the atmosphere of multi-choice transportation parameters, the mathematical model of MCMTP is defined as follows:

Model M1

$$
\begin{aligned}
\min : Z^{t} & =\sum_{i=1}^{m} \sum_{j=1}^{n}\left(\tilde{C}_{i j}^{t 1} \text { or } \tilde{C}_{i j}^{t 2} \text { or } \cdots \text { or } \tilde{C}_{i j}^{t r}\right) x_{i j}, \quad t=1,2, \ldots, K \\
\text { subject to } \quad \sum_{j=1}^{n} x_{i j} & \leq \tilde{a}_{i}^{1} \text { or } \tilde{a}_{i}^{2} \text { or } \cdots \text { or } \tilde{a}_{i}^{p}, \quad i=1,2, \ldots, m \\
\sum_{i=1}^{m} x_{i j} & \geq \tilde{b}_{j}^{1} \text { or } \tilde{b}_{j}^{2} \text { or } \cdots \text { or } \tilde{b}_{j}^{q}, \quad j=1,2, \ldots, n \\
x_{i j} & \geq 0, \forall i \text { and } j .
\end{aligned}
$$

Here $\left(\tilde{C}_{i j}^{t 1}\right.$ or $\tilde{C}_{i j}^{t 2}$ or $\cdots$ or $\left.\tilde{C}_{i j}^{t r}\right),\left(\tilde{a}_{i}^{1}\right.$ or $\tilde{a}_{i}^{2}$ or $\cdots$ or $\left.\tilde{a}_{i}^{p}\right)$, and $\left(\tilde{b}_{j}^{1}\right.$ or $\tilde{b}_{j}^{2}$ or $\cdots$ or $\left.\tilde{b}_{j}^{q}\right)$ are the multi-choice cost, supply, and demand parameters for the $t$ th objective function, respectively. In a objective function, respectively. In a transportation problem, the total demand should be less or equal to the total capacity of supply to get a feasible solution. In the present case for multi-choice of supply and demands, the information of total capacity of supply in the origins and demands in the destinations is not precisely calculated. So we have selected here the maximum possible supply in the origins and consequently the minimum demand in the destinations and then formulated the feasibility condition as $\sum_{i=1}^{m} \max \left\{\tilde{a}_{i}^{1}, \tilde{a}_{i}^{2}, \ldots, \tilde{a}_{i}^{p}\right\} \geq \sum_{j=1}^{n} \min \left\{\tilde{b}_{j}^{1}, \tilde{b}_{j}^{2}, \ldots, \tilde{b}_{j}^{q}\right\}$. This feasibility condition is the best possible wide range of feasible region of the MCMTP. However, the feasibility condition can be remodeled as per as decision maker's choice.

\section{Transformation technique for multi-choice parameters like cost, supply, and demand to the equivalent form}

When there are multiple choice of parameters such as cost, supply, and demand, we should select a single choice satisfying supply and demand restrictions. The selection of choices should be done in such a way that the whole problem is optimized. Introduction of binary variables is an important concept to select a choice in the problem.

If we have to choose one among $t$ number of possibilities, then we use $p$ number of binary variables where $2^{p-1}<t \leq 2^{p}$. Let $t={ }^{p} C_{0}+{ }^{p} C_{1}+{ }^{p} C_{2}+\cdots+{ }^{p} C_{d}+k$, for some $d$ satisfying $1 \leq d \leq p, 0 \leq k<^{p} C_{d+1}$. Let us take $p$ binary variables $z_{j}^{1}, z_{j}^{2}, \ldots, z_{j}^{p}$ to deduce a formula which will select one among the $t$ values $c_{j}^{1}, c_{j}^{2}, \ldots, c_{j}^{t}$. Let us form a function with $p$ binary variables, $f_{0}(z)=\left(z_{j}^{1} z_{j}^{2} \ldots z_{j}^{p}\right) c_{j}^{1}$ where $z=\left(z_{j}^{1}, z_{j}^{2}, \ldots, z_{j}^{p}\right)$. When each $z_{j}^{i}=1$ for $i=1,2, \ldots p, f_{0}(z)=c_{j}^{1}$. Thus, $f_{0}(z)=c_{j}^{1}$, when $z_{j}^{1}+z_{j}^{2}+\cdots+z_{j}^{p}=p$. Again, let us assume a function

$$
f_{1}(z)=\left(1-z_{j}^{1}\right) z_{j}^{2} \ldots z_{j}^{p} c_{j}^{2}+\left(1-z_{j}^{2}\right) z_{j}^{1} z_{j}^{3} \ldots z_{j}^{p} c_{j}^{3}+\cdots+\left(1-z_{j}^{p}\right) z_{j}^{1} \ldots z_{j}^{p-1} c_{j}^{1+{ }^{p} C_{1}}
$$


When $z_{j}^{1}+z_{j}^{2}+\cdots+z_{j}^{p}=p-1, f_{1}(z)$ gives output one of the following $c_{j}^{t} \mathrm{~s}$ : $c_{j}^{2}, c_{j}^{3}, \ldots, c_{j}^{1+{ }^{p} C_{1}}$. Similarly, we consider

$f_{2}(z)=\left(1-z_{j}^{1}\right)\left(1-z_{j}^{2}\right) z_{j}^{3} \ldots z_{j}^{p} c_{j}^{1+{ }^{p} C_{1}+1}+\left(1-z_{j}^{1}\right)\left(1-z_{j}^{3}\right) z_{j}^{2} z_{j}^{4} \ldots z_{j}^{p} c_{j}^{1+{ }^{p} C_{1}+2}+\cdots+$ $\left(1-z_{j}^{1}\right)\left(1-z_{j}^{p}\right) z_{j}^{2} \ldots z_{j}^{p-1} c_{j}^{1+{ }^{p} C_{1}+(p-2)}+\left(1-z_{j}^{2}\right)\left(1-z_{j}^{3}\right) z_{j}^{1} z_{j}^{4} \ldots z_{j}^{p} c_{j}^{1+{ }^{p} C_{1}+(p-2)+1}+$

$+\left(1-z_{j}^{p-1}\right)\left(1-z_{j}^{p}\right) z_{j}^{1} \ldots z_{j}^{p-2} c_{j}^{1+{ }^{p} C_{1}+{ }^{p} C_{2}}$

When $z_{j}^{1}+z_{j}^{2}+\cdots+z_{j}^{p}=p-2$, the above function $f_{2}(z)$ gives one among the following $c_{j}^{t} \mathrm{~s}: c_{j}^{1+{ }^{p} C_{1}+1}, c_{j}^{1+{ }^{p} C_{1}+2}, \ldots, c_{j}^{1+{ }^{p} C_{1}+{ }^{p} C_{2}}$.

Proceeding in the same manner, we find

$f_{d}(z)=\left(1-z_{j}^{1}\right)\left(1-z_{j}^{2}\right) \cdots\left(1-z_{j}^{d}\right) z_{j}^{d+1} \cdots z_{j}^{p} c_{j}^{1+{ }^{p} C_{1}+{ }^{p} C_{2}+\cdots+{ }^{p} C_{d-1}+1}$

$+\left(1-z_{j}^{1}\right)\left(1-z_{j}^{2}\right) \cdots\left(1-z_{j}^{d-1}\right)\left(1-z_{j}^{d+1}\right) z_{j}^{d} z_{j}^{d+2} \cdots z_{j}^{p} c_{j}^{1+{ }^{p} C_{1}+{ }^{p} C_{2}+\cdots+{ }^{p} C_{d-1}+2}+$

$+\left(1-z_{j}^{p-d+1}\right)\left(1-z_{j}^{p-d+2}\right)\left(1-z_{j}^{p}\right) z_{j}^{1} \ldots z_{j}^{p-d} c_{j}^{1+{ }^{p} C_{1}+{ }^{p} C_{2}+\cdots+{ }^{p} C_{d}}$

When $z_{j}^{1}+z_{j}^{2}+\cdots+z_{j}^{p}=p-d$, the above function gives one among the following $c_{j}^{t} \mathrm{~s}: c_{j}^{1+{ }^{p} C_{1}+{ }^{p} C_{2}+\ldots{ }^{p} C_{d-1}+1}, c_{j}^{1+{ }^{p} C_{1}+{ }^{p} C_{2}+\ldots{ }^{p} C_{d-1}+2}, \ldots, c_{j}^{1+{ }^{p} C_{1}+{ }^{p} C_{2}+\ldots{ }^{p} C_{d-1}+{ }^{p} C_{d}}$.

When $k=0$, the function $f(z)=f_{0}(z)+f_{1}(z)+\cdots+f_{d}(z)$ gives one of the value $c_{j}^{t}$ for all $z$ satisfying $p-d \leq z_{j}^{1}+z_{j}^{2}+\cdots+z_{j}^{p} \leq p$.

If $k \neq 0$, then $k<^{p} C_{d+1}$ and we formulate the function

$$
\begin{aligned}
f_{d+1}(z)= & \left(1-z_{j}^{i_{1}}\right)\left(1-z_{j}^{i_{2}}\right) \ldots\left(1-z_{j}^{i_{d}}\right)\left(1-z_{j}^{i_{d+1}}\right) z_{j}^{d+2} \ldots z_{j}^{n} c_{j}^{t-k+1} \\
& +\left(1-z_{j}^{i_{1}}\right)\left(1-z_{j}^{i_{2}}\right) \ldots\left(1-z_{j}^{i_{d}}\right)\left(1-z_{j}^{i_{d+2}}\right) z_{j}^{d+1} z_{j}^{d+3} \ldots z_{j}^{p} c_{j}^{t-k+2} \\
& +\cdots+\left(\text { terms up to } c_{j}^{t}\right)
\end{aligned}
$$

When $z_{j}^{1}+z_{j}^{2}+\cdots+z_{j}^{p}=p-(d+1), f_{d+1}(z)$ can give one output among ${ }^{p} C_{d+1}$ number of choices. Here we have used ${ }^{p} C_{d+1}-k$ restrictions to restrict its possible outputs in $k$ numbers. Let the $k$ th term occur at $i_{1}=i_{1}^{\prime}, i_{2}=i_{2}^{\prime}, \ldots, i_{d+1}=i_{d+1}^{\prime}$, then the restrictions are

$p-(d+1) \leq z_{j}^{i_{1}}+z_{j}^{i_{2}}+\cdots+z_{j}^{i_{p}} \leq p$

$z_{j}^{i_{1}}+z_{j}^{i_{2}}+\cdots+z_{j}^{i_{d+1}} \geq 1$, for all $i_{1}=i_{1}^{\prime}, i_{2}=i_{2}^{\prime}, \ldots i_{d}=i_{d}^{\prime}, i_{p} \geq i_{d+1}>i_{d+1}^{\prime}$;

$z_{j}^{i_{1}}+z_{j}^{i_{2}}+\cdots+z_{j}^{i_{d+1}} \geq 1$, for all $i_{1}=i_{1}^{\prime}, i_{2}=i_{2}^{\prime}, \ldots, i_{d-1}=i_{d-1}^{\prime}, i_{p-1} \geq i_{d}>i_{d}^{\prime}$;

$\vdots$

$z_{j}^{i_{1}}+z_{j}^{i_{2}}+\cdots+z_{j}^{i_{d+1}} \geq 1$, for all $i_{p-d-1} \geq i_{1}>i_{1}^{\prime}$.

Thus, $f(z)=f_{0}(z)+f_{1}(z)+\cdots+f_{d}(z)+f_{d+1}(z)$ gives the generalized selection function of the multi-choice $c_{j}^{t}$ s.

Without loss of any generality in treating the value of $c_{j}^{t}=1$ and using the product and summation notation, we have formulated the following formulae to select the crisp value of multi-choice parameters:

$$
\begin{array}{r}
\prod_{i=1}^{p} z_{j}^{i}+\sum_{i_{1}=1}^{p}\left[\left(1-z_{j}^{i_{1}}\right) \prod_{i=1, i \neq i_{1}}^{p} z_{j}^{i}\right]+\sum_{i_{2}=2 i_{2}>i_{1}}^{p} \sum_{i_{1}=1}^{p}\left[\left(1-z_{j}^{i_{1}}\right)\left(1-z_{j}^{i_{2}}\right) \prod_{i=1\left(i \neq i_{1}, i_{2}\right)}^{p} z_{j}^{i}\right] \\
+\cdots+\sum_{\substack{i_{d}=d \\
i_{d}>i_{(d-1)}}}^{p} \sum_{\substack{i_{d-1}=d-1 \\
i_{d-1}>i_{d-2}}}^{p} \ldots \sum_{i_{1}=1}^{p}\left[\left(1-z_{j}^{i_{1}}\right)\left(1-z_{j}^{i_{2}}\right) \ldots\left(1-z_{j}^{i_{d}}\right) \prod_{\substack{i=1, i \neq\left(i_{1} \ldots, i_{d}\right) \\
z_{j}}}^{z_{j}^{i}}\right.
\end{array}
$$


where $p-d \leq z_{j}^{i_{1}}+z_{j}^{i_{2}}+\cdots+z_{j}^{i_{p}} \leq p$ for all $i_{1}<i_{2}<\cdots<i_{p}$.

When $k \neq 0$, we add first $k$ terms with the above function from the following formula:

$$
\begin{gathered}
\left(1-z_{j}^{i_{1}}\right)\left(1-z_{j}^{i_{2}}\right) \ldots\left(1-z_{j}^{i_{d}}\right)\left(1-z_{j}^{i_{d+1}}\right) \prod_{i=1, i \neq\left(i_{1}, . . i_{d}, i_{d+1}\right)}^{p} z_{j}^{i} \\
+\left(1-z_{j}^{i_{1}}\right)\left(1-z_{j}^{i_{2}}\right) \ldots\left(1-z_{j}^{i_{d}}\right)\left(1-z_{j}^{i_{d+2}}\right) \prod_{i=1, i \neq\left(i_{1}, . . i_{d}, i_{d+2}\right)}^{p} z_{j}^{i} \\
+\cdots+\left(1-z_{j}^{i_{1}}\right)\left(1-z_{j}^{i_{2}}\right) \ldots\left(1-z_{j}^{i_{d}}\right)\left(1-z_{j}^{i_{p}}\right) \prod_{i=1, i \neq\left(i_{1}, . . i_{d}, i_{p}\right)}^{p} z_{j}^{i} \\
+\left(1-z_{j}^{i_{1}}\right)\left(1-z_{j}^{i_{2}}\right) \ldots\left(1-z_{j}^{i_{d+1}}\right)\left(1-z_{j}^{i_{d+2}}\right) \prod_{i=1, i \neq\left(i_{1}, . . i_{d+1}, i_{d+2}\right)}^{p} z_{j}^{i} \\
+\left(1-z_{j}^{i_{1}}\right)\left(1-z_{j}^{i_{2}}\right) \ldots\left(1-z_{j}^{i_{d+1}}\right)\left(1-z_{j}^{i_{d+3}}\right) \prod_{i=1, i \neq\left(i_{1}, . ., i_{d+1}\right)}^{p} z_{j}^{i}+ \\
+\left(1-z_{j}^{i_{p-(d+1)}}\right)\left(1-z_{j}^{i_{p-(d-1)}}\right) \ldots\left(1-z_{j}^{i_{p-1}}\right)\left(1-z_{j}^{i_{p}}\right) \prod_{i \neq\left(i_{p-d-1}, i_{p-d+1} \ldots, i_{p}\right)}^{p} z_{j}^{i}
\end{gathered}
$$

Assuming that $i_{1}<i_{2}<\cdots<i_{p}$ and let $k$ th term occurred at $i_{1}^{\prime}, i_{2}^{\prime}, \ldots, i_{d+1}^{\prime}$, then the restrictions are

$p-(d+1) \leq z_{j}^{i_{1}}+z_{j}^{i_{2}}+\cdots+z_{j}^{i_{p}} \leq p$

$z_{j}^{i_{1}}+z_{j}^{i_{2}}+\cdots+z_{j}^{i_{d+1}} \geq 1$, for all $i_{1}=i_{1}^{\prime}, i_{2}=i_{2}^{\prime}, \ldots i_{d}=i_{d}^{\prime}, i_{p} \geq i_{d+1}>i_{d+1}^{\prime}$;

$z_{j}^{i_{1}}+z_{j}^{i_{2}}+\cdots+z_{j}^{i_{d+1}} \geq 1$, for all $i_{1}=i_{1}^{\prime}, i_{2}=i_{2}^{\prime}, \ldots, i_{d-1}=i_{d-1}^{\prime}, i_{p-1} \geq i_{d}>i_{d}^{\prime}$;

$z_{j}^{i_{1}}+z_{j}^{i_{2}}+\cdots+z_{j}^{i_{d+1}} \geq 1$, for all $i_{p-d-1} \geq i_{1}>i_{1}^{\prime}$.

$$
\text { Let } \tilde{C}_{i j}^{t}=\sum_{g=1}^{T}(\text { term })^{g} C_{i j}^{t^{g}} i=1,2, \ldots, m ; j=1,2, \ldots, n
$$

where (term) ${ }^{g}$ (for $g=1,2, \ldots, T$ ) are the $T$ number of terms in the functions of the binary variables mentioned in above. Similarly,

$$
\tilde{a}_{i}=\sum_{g=1}^{P}(\text { term })^{g} a_{i}^{g} i=1,2, \ldots, m
$$

and $\tilde{b}_{j}=\sum_{g=1}^{Q}(\text { term })^{g} b_{j}^{g} j=1,2, \ldots, n$

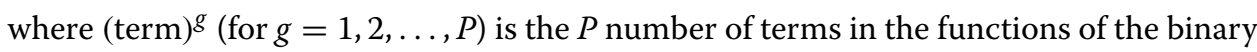
variables mentioned above to reduce the $P$ number of choices $a_{i}^{g}$ to single choice $a_{i}^{\prime}$, and (term) ${ }^{g}$ (for $g=1,2, \ldots, Q$ ) is the $Q$ number of terms in functions of binary variables mentioned above to reduce the $Q$ number of choices $b_{j}^{g}$ to single choice $b_{j}^{\prime}$. 


\section{Reduction of MCMTP to MOTP}

The MCMTP as given in the Model M1 transformed to a MOTP by transforming the multi-choice parameters in the objective functions (5) and the multi-choice supplies and demands in constraints (6) and (7) to single-valued ones, using the technique described in subsection "Transformation technique for multi-choice parameters like cost, supply, and demand to the equivalent form". Thus, the equivalent MOTP of Model M1 is given in the following model:

Model M2

$$
\begin{aligned}
& \min : Z^{t}=\sum_{i=1}^{m} \sum_{j=1}^{n} C_{i j}^{\prime t} x_{i j}, \quad t=1,2, \ldots, K \\
& \text { subject to } \sum_{j=1}^{n} x_{i j} \leq a_{i}^{\prime}, \quad i=1,2, \ldots, m \\
& \sum_{i=1}^{n} x_{i j} \geq b_{j}^{\prime}, j=1,2, \ldots, n \\
& \text { and } x_{i j} \geq 0, \quad \forall i \text { and } j
\end{aligned}
$$

Here $C_{i j}^{\prime t}, a_{i}^{\prime}, b_{j}^{\prime}$ are the reduced cost, supply, and demand parameters of $t$ th objective function in MOTP, respectively, and $\sum_{i=1}^{m} \max \left\{\tilde{a}_{i}^{1}, \tilde{a}_{i}^{2}, \ldots, \tilde{a}_{i}^{p}\right\} \geq \sum_{j=1}^{n}$ $\min \left\{\tilde{b}_{j}^{1}, \tilde{b}_{j}^{2}, \ldots, \tilde{b}_{j}^{q}\right\}$ is the feasibility condition.

The transportation problem Model M2 is same as the problem described in Model 1. We can solve the Model M2 as the procedure described using the different techniques to solve Model 1.

\section{Solution procedure}

The approaches such as goal programming and revised multi-choice goal programming are used to solve the MOTP, which are defined as follows:

\section{A. Goal programming approach}

Let us briefly discuss the goal programming approach for solving MOTP (see Model $1 A$ ). If $d_{t}^{+}$and $d_{t}^{-}$be positive and negative deviations corresponding to the $t$ th goal of the objective function, then the mathematical model is defined as follows:

Model $1 A$

$$
\begin{array}{r}
\min \sum_{t=1}^{K} w_{t}\left(d_{t}^{+}+d_{t}^{-}\right) \\
\text {subject to } Z^{t}(X)-d_{t}^{+}+d_{t}^{-}=y_{t}, \quad t=1,2, \ldots, K \\
g_{t, \min } \leq y_{t} \leq g_{t, \max }, \quad t=1,2, \ldots, K \\
d_{t}^{+}, d_{t}^{-} \geq 0, \quad t=1,2, \ldots, K
\end{array}
$$

$$
\text { and (2) to (4) }
$$

\section{B. Revised multi-choice goal programming approach}

In the similar way, the RMCGP is introduced to solve the MOTP. Let us assume that the multiple goals are considered to the objective functions and this can be achieved by considering the following model (see Model 1B) as 
Model 1B

$$
\begin{array}{r}
\min \sum_{t=1}^{K} w_{t}\left(d_{t}^{+}+d_{t}^{-}\right)+\alpha_{t}\left(e_{t}^{+}+e_{t}^{-}\right) \\
\text {subject to } Z^{t}(X)-d_{t}^{+}+d_{t}^{-}=y_{t}, \quad t=1,2, \ldots, K \\
y_{t}-e_{t}^{+}+e_{t}^{-}=g_{t, \max }, \text { or } g_{t, \min } \quad t=1,2, \ldots, K \\
g_{t, \min } \leq y_{t} \leq g_{t, \max }, \quad t=1,2, \ldots, K \\
d_{t}^{+}, d_{t}^{-}, e_{t}^{+}, e_{t}^{-} \geq 0, \quad t=1,2, \ldots, K
\end{array}
$$

and (2) to (4)

where $t$ th aspiration level is defined as $y_{t}$ which is the continuous variable that lies between the upper $\left(g_{t, \max }\right)$ and lower $\left(g_{t, \min }\right)$ bounds. Again, $e_{t}^{+}$and $e_{t}^{-}$are positive and negative deviations attached to $t$ th goal of $\left|y_{t}-g_{t, \max }\right|$, and $\alpha_{t}$ is the weight attached to the sum of the deviations of $\left|y_{t}-g_{t, \max }\right|$.

\section{Utility function approach to solve MOTP}

Here, the concept of utility function has been addressed to solve MOTP. A short introduction is presented here and then we discuss the methodology for solving MOTP using utility function.

\section{Utility function}

In this paper, introduction of utility is taken to be correlative to 'Desire' or 'Want'. It has been already argued that desire cannot be measured directly, but only indirectly, by the outward phenomena in which the context is presented.

Definition 1. The utility function describes a function $U: X \longrightarrow \Re$ which assigns a real number to every outcome in such a way that it captures DM's preferences over the desired goals of the objectives, where $X$ is the set of feasible points and $\Re$ is the set of real numbers.

The purpose of this study is to derive the achievement function of MOTP under the light of utility function for the DM according to the priority of goals. In our proposed approach, the DM wants to maximize his/her expected utility. For the sake of simplicity, two popular utility functions (linear and S-shaped) are considered as follows.

Linear utility function $u_{i}\left(y_{i}\right)$ for decision-making (management) problems can be found in Lai and Hwang [14] and S-shaped utility function (for the same purpose) has been proposed by Chang [15]. The utility function is generally considered in three cases as follows:

Case 1: Left linear utility function (LLUF)

$$
u_{i}\left(y_{i}\right)= \begin{cases}1, & \text { if } y_{i} \leq g_{i, \min } \\ \frac{g_{i, \max }-y_{i}}{g_{i, \max }-g_{i, \min },} & \text { if } g_{i, \min } \leq y_{i} \leq g_{i, \max }, i=1,2, \ldots, K \\ 0, & \text { if if } y_{i} \geq g_{i, \max }\end{cases}
$$

Case 2: Right linear utility function (RLUF)

$$
u_{i}\left(y_{i}\right)= \begin{cases}1, & \text { if } y_{i} \geq g_{i, \max } \\ \frac{y_{i}-g_{i \min }}{g_{i, \max }-g_{i, \min },}, & \text { if } g_{i, \min } \leq y_{i} \leq g_{i, \max }, i=1,2, \ldots, K \\ 0, & \text { if if } y_{i} \leq g_{i, \min }\end{cases}
$$


Case 3: S-shaped utility function

$$
u_{i}\left(y_{i}\right)=\left\{\begin{array}{ll}
0, & \text { if } y_{i} \leq g_{i 2} \\
\frac{y_{i}-g_{i 2}}{g_{i 8}-g_{i 2}}, & \text { if } g_{i 2} \leq y_{i} \leq g_{i 4} \\
\frac{y_{i}-g_{i 3}}{g_{i 6}-g_{i 3}}, & \text { if } g_{i 4} \leq y_{i} \leq g_{i 5} \\
\frac{y_{i}-g_{i 1}}{g_{i 7}-g_{i 1}}, & \text { if } g_{i 5} \leq y_{i} \leq g_{i 7}
\end{array}, i=1,2, \ldots, K\right.
$$

where $g_{i, \min }$ and $g_{i, \max }$ are lower and upper bounds corresponding to the $i$ th goal respectively. The graphs of above utility functions are drawn in the following figures (see Figures 1,2 and 3).

\section{Model formulation for case 1}

The DM would like to increase the utility value $u_{t}\left(y_{t}\right)$ as much as possible in the case of LLUF (Figure 1). In order to achieve this goal, the value of $y_{t}$ should be as close to the target value $g_{t, \text { min }}$ as possible. The MOTP from Model 1A can be reformulated using the proposed LLUF as follows:

Model 2A

$$
\min \sum_{t=1}^{K} w_{t}\left(d_{t}^{+}+d_{t}^{-}\right)+\beta_{t} f_{t}^{-}
$$

subject to

$$
\begin{array}{r}
Z^{t}(X)-d_{t}^{+}+d_{t}^{-}=y_{t}, \quad t=1,2, \ldots, K \\
g_{t, \min } \leq y_{t} \leq g_{t, \max }, \quad t=1,2, \ldots, K \\
u_{t} \leq \frac{g_{t, \max }-y_{t}}{g_{t, \max }-g_{t, \min }}, \quad t=1,2, \ldots, K \\
u_{t}+f_{t}^{-}=1, \quad t=1,2, \ldots, K \\
u_{t}, f_{t}^{-} \geq 0, \quad t=1,2, \ldots, K
\end{array}
$$

$$
\text { and (2) to (4) }
$$

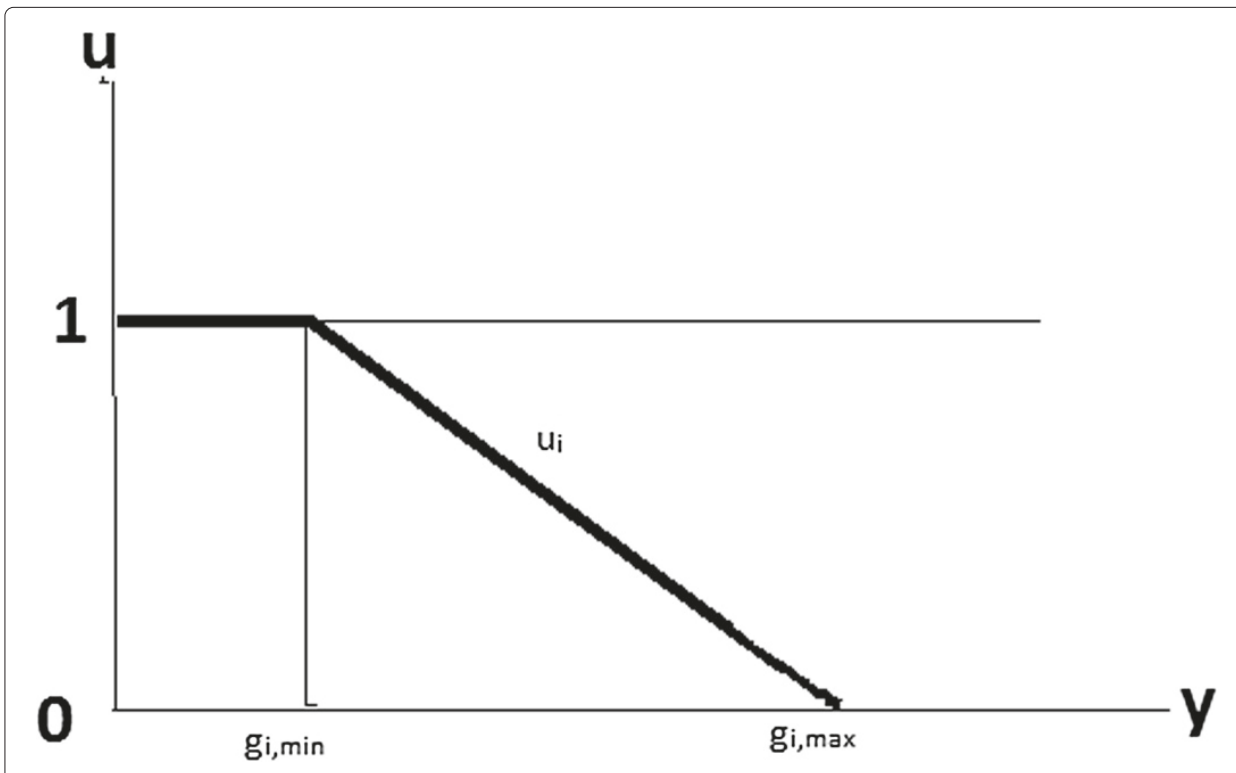

Figure 1 LLUF. 


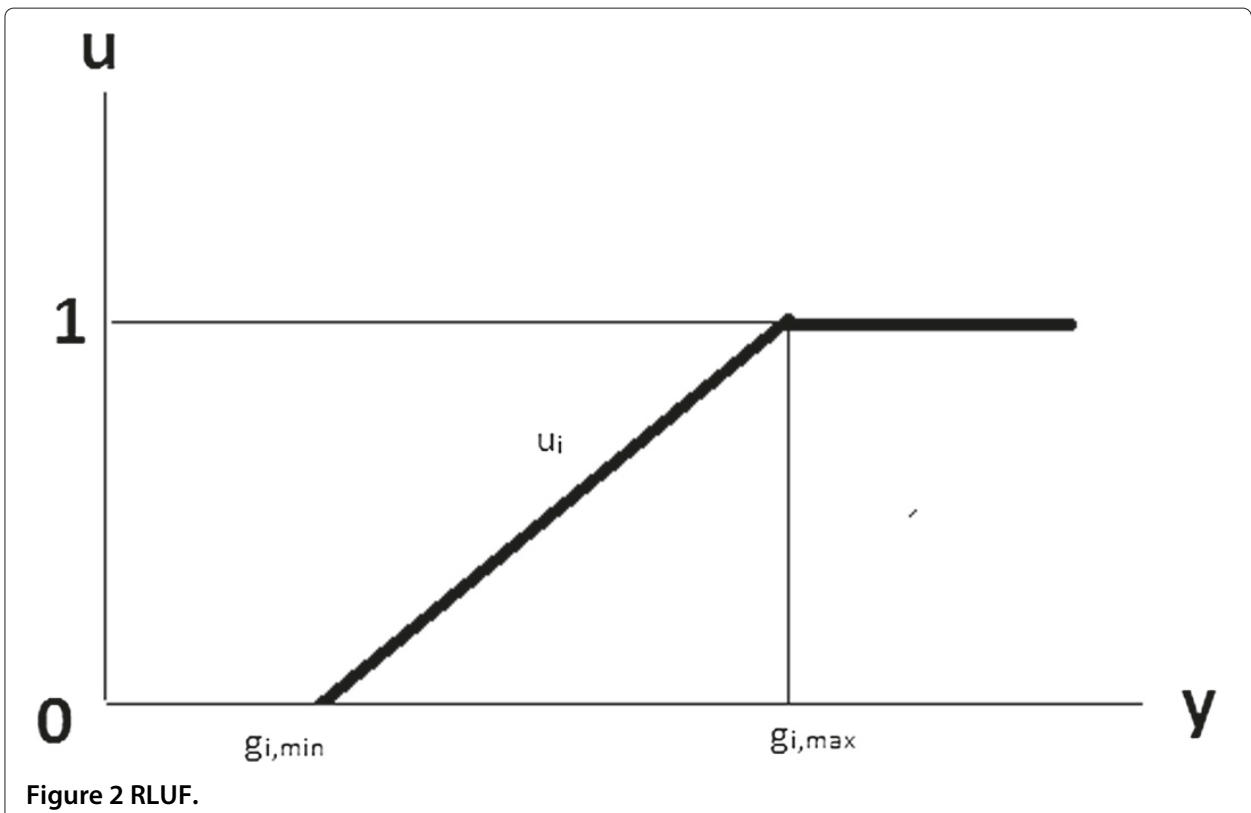

where $\beta_{t}$ is the weight attached to deviation $f_{t}^{-}$. The role of weight $\beta_{t}$ can be seen as the preferential component for the utility value $u_{t}$.

Proposition 1. Achievement of optimal utility in the LLUF (Figure 1) is equivalent to the optimal solution of Model $2 A$.

Proof. When $u_{t}$ approaches to the highest value 1, then the deviation $f_{t}^{-} \rightarrow 0$ of the utility function (from Equation 29), because $f_{t}^{-}$should be minimized in the objective function to obtain the optimal solution of Model 2A. This represents $y_{t}$ approach to $g_{t, \text { min }}$ (from Equation 28), and $Z_{t}(X)$ is also closer to $g_{t, \text { min }}$ (from Equation 26) because $d_{t}^{+}$and

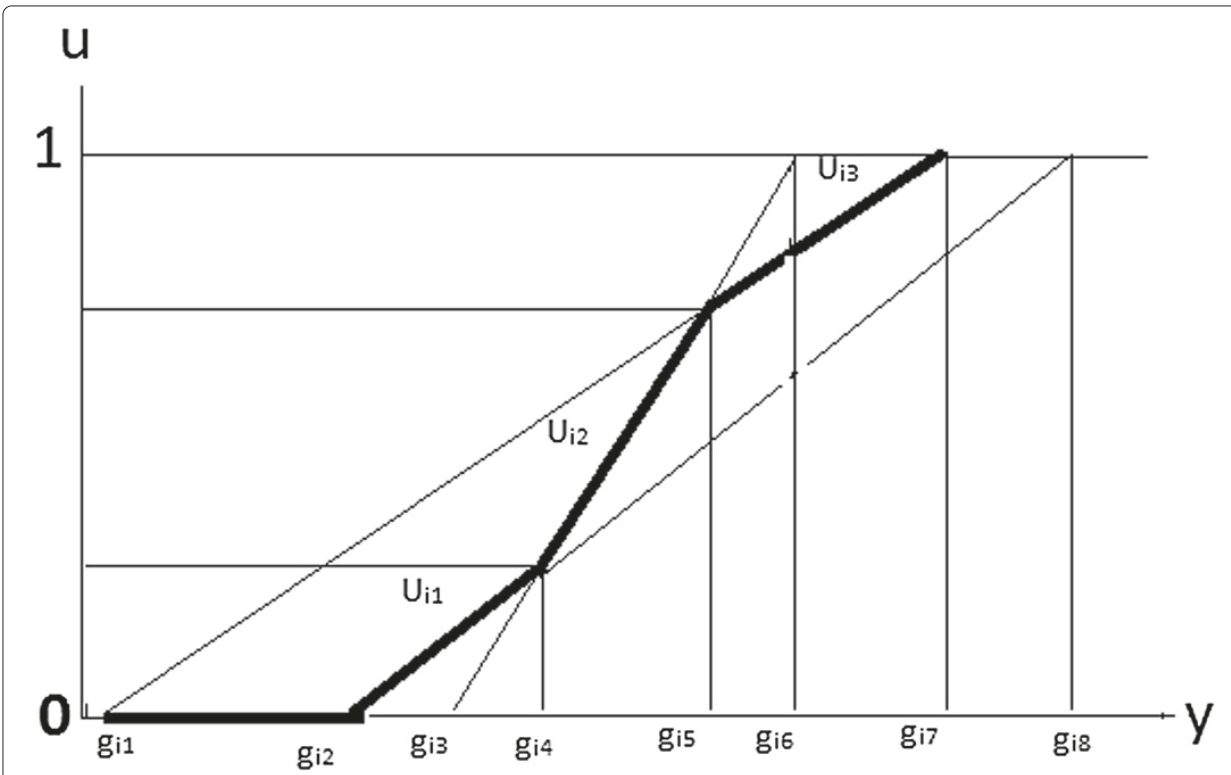

Figure 3 S-shaped utility function. 
$d_{t}^{-}$should also be minimized in the objective function. It is obvious that the behavior of Model 2A and the level of utility are achieved. This completes the proof.

\section{Model formulation for case 2}

The DM would like to increase the utility value $u_{t}\left(y_{t}\right)$ as much as possible in the case of RLUF (Figure 2). In order to achieve this goal, the value of $y_{t}$ should be as close to the target value $g_{t, \max }$ as possible. The MOTP from Model $1 \mathrm{~A}$ can be reformulated using the proposed RLUF as follows:

$$
\begin{array}{r}
\text { Model } 2 B \quad \min \sum_{t=1}^{K} w_{t}\left(d_{t}^{+}+d_{t}^{-}\right)+\beta_{t} f_{t}^{-} \\
\text {subject to } Z^{t}(X)-d_{t}^{+}+d_{t}^{-}=y_{t}, \quad t=1,2, \ldots, K \\
g_{t, \min } \leq y_{t} \leq g_{t, \max }, \quad t=1,2, \ldots, K \\
u_{t} \leq \frac{y_{t}-g_{t, \min }}{g_{t, \max }-g_{t, \min }} \\
u_{t}+f_{t}^{-}=1 \\
u_{t}, f_{t}^{-} \geq 0 \\
\text { and }(2) \text { to }(4)
\end{array}
$$

where $\beta_{t}$ is the weight attached to the deviation $f_{t}^{-}$. The role of weight $\beta_{t}$ can be seen as a preferential component for the utility value $u_{t}$.

Proposition 2. Achievement of optimal utility in the RLUF (Figure 2) is equivalent to the optimal solution of Model $2 B$.

Proof. Similar way can be followed as we have done in Proposition 1.

The advantages of the use of LLUF and RLUF in the decision-making problems are as follows:

(1) The DM can easily formulate their MOTP by taking into account their preference mappings with utility functions in real situation.

(2) The two linear utility models represented as linear form which can be easily solved using software.

Due to variation of deviation variables $d_{t}^{+}, d_{t}^{-}$, and $f^{-}$in different ranges, biasness may occur towards the objective functions with larger magnitude. Normalization technique may help to remove this biasness. Several normalization approaches such as percentage, Euclidean, summation, and zero-one notarizations (Tamiz et al. [12]; Kettani et al. [16]) are available to execute this. According to the normalization technique proposed by Tamiz et al. [12], Model 2A can be redesigned as follows:

$$
\begin{array}{r}
\min \sum_{t=1}^{K}\left[\frac{w_{t}\left(d_{t}^{+}+d_{t}^{-}\right)+\beta_{t} f_{t}^{-}}{\phi_{t}}\right] \\
\text { subject to (26) to (30) and (2) to (4) }
\end{array}
$$

where $\phi_{t}$ is the normalization constant for $t$ th goal. 
In order to solve this problem, utility normalization concept is introduced as follows: Let $d_{t}^{+}, d_{t}^{-} \in\left[0, \bar{u}_{t}\right]$ and $f_{t}^{-} \in[0,1]$ where $\bar{u}_{t}$ is the upper bound of $d_{t}^{+}$and $d_{t}^{-}$. The normalized weights $w_{t}$ and $\beta_{t}$ can be easily obtained as $w_{t}=\frac{1}{1+\bar{u}_{t}}$ and $\beta_{t}=\frac{\bar{u}_{t}}{1+\bar{u}_{t}}$. This technique of normalization ensures that deviation variables $d_{t}^{+}, d_{t}^{-}$, and $f_{t}^{-}$approximated the same magnitude. Similarly, the same methodology can be applied to the Model $2 B$.

The utility value for S-shaped utility function can be expressed as a sum of linear utility functions (RLUF or LLUF) by introducing binary variables [17]. But Chang [15] proposed in his paper that the utility value for S-shaped utility function can be considered without using the binary variables and this is shown in the following model (see Model 2C):

Model 2C

$$
\begin{aligned}
& \min \sum_{t=1}^{K} w_{t}\left[p_{t 1}+p_{t 2}+p_{t 3}\right]+\beta_{t} f_{t}^{-} \\
& \text {subject to } Z^{t}(X)-d_{t}^{+}+d_{t}^{-}=y_{t}, \quad t=1,2, \ldots, K \\
& g_{t, \min } \leq y_{t} \leq g_{t, \max }, t=1,2, \ldots, K, \\
& u_{t}=\left[u_{t}\left(g_{t 4}\right)-u_{t}\left(g_{t 2}\right)\right] \frac{p_{t 1}-p_{t 2}}{g_{t 4}-g_{t 2}}+\left[u_{t}\left(g_{t 5}\right)-u_{t}\left(g_{t 4}\right)\right] \frac{p_{t 2}-p_{t 3}}{g_{t 5}-g_{t 4}} \\
& +\left[u_{t}\left(g_{t 7}\right)-u_{t}\left(g_{t 5}\right)\right] \frac{p_{t 3}}{g_{t 7}-g_{t 5}}, t=1,2, \ldots, K \\
& y_{t}-p_{t 1}+n_{t 1}=g_{t 2}, t=1,2, \ldots, K \\
& y_{t}-p_{t 2}+n_{t 2}=g_{t 4}, t=1,2, \ldots, K \\
& y_{t}-p_{t 3}+n_{t 3}=g_{t 5}, t=1,2, \ldots, K \\
& u_{t}+f_{t}^{-}=1, t=1,2, \ldots, K \\
& u_{t}, p_{t l}, n_{t l} \geq 0, t=1,2, \ldots, K, l=1,2,3 \\
& \text { and (2) to (4) }
\end{aligned}
$$

MCMTP which occurred in many real-life situations can be reduced to MOTP and then the problem can be reduced to the models such as $2 \mathrm{~A}, 2 \mathrm{~B}$, and $2 \mathrm{C}$, with interval goals under the consideration of utility functions related to these goals. Solving the formulated problem, the DM obtained the satisfactory solution.

\section{Numerical examples}

Here we have presented two numerical examples; the first one explores the applicability of MOTP and the second one represents the applicability of MCMTP.

\section{Case 1}

Let us consider the following MOTP (see Model 3) with three objectives:

Model 3

Goal $1: Z^{1}=7 x_{11}+8 x_{12}+7.5 x_{13}+8 x_{21}+7.2 x_{22}+8.4 x_{23}+9 x_{31}+8 x_{32}+7.7 x_{33}$ with goal as [170, 220], more is better, follows RLUF.

Goal $2: Z^{2}=50 x_{11}+65 x_{12}+62 x_{13}+60 x_{21}+55 x_{22}+58 x_{23}+65 x_{31}+60 x_{32}+58 x_{33}$ with goal as $[1,550 ; 1,800]$, less is better, follows LLUF.

Goal 3: $Z^{3}=10 x_{11}+8 x_{12}+9 x_{13}+8.5 x_{21}+9.5 x_{22}+8.5 x_{23}+9.5 x_{31}+8.8 x_{32}+9 x_{33}$ with goal as $[200,290]$, more is better, follows $S$-shaped utility function as given in Figure 4. 


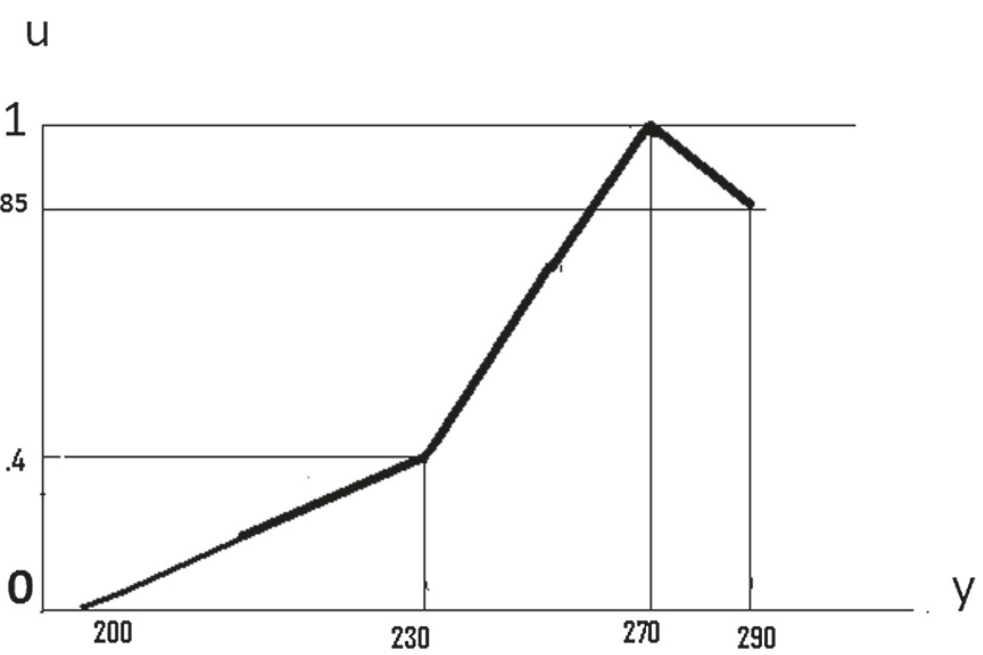

Figure 4 S-shaped utility function for the goal 3 of the proposed example.

$$
\begin{aligned}
& \text { subject to } x_{11}+x_{12}+x_{13} \leq 10 \\
& x_{21}+x_{22}+x_{23} \leq 9 \\
& x_{31}+x_{32}+x_{33} \leq 11 \\
& x_{11}+x_{21}+x_{31} \geq 9 \\
& x_{12}+x_{22}+x_{32} \geq 8 \\
& x_{13}+x_{23}+x_{33} \geq 10 \\
& x_{i j} \geq 0, \quad \forall i, j=1,2,3
\end{aligned}
$$

To achieve the goals in the proposed problem (see Model 3), we may formulate the following models.

In the proposed problem, the deviations of goals 1, 2, 3 are 50, 250, 90, respectively. By considering the weights $w_{1}=\frac{1}{50}, w_{2}=\frac{1}{250}, w_{3}=\frac{1}{90}$ for the Model 1A, the above Model 3 reduces to the following model (see Model $3 A$ ) as

Model 3A

$$
\begin{array}{r}
\min \frac{1}{50}\left(d_{1}^{+}+d_{1}^{-}\right)+\frac{1}{250}\left(d_{2}^{+}+d_{2}^{-}\right)+\frac{1}{90}\left(d_{3}^{+}+d_{3}^{-}\right) \\
\text {subject to } Z^{1}=7 x_{11}+8 x_{12}+7.5 x_{13}+8 x_{21}+7.2 x_{22}+8.4 x_{23} \\
+9 x_{31}+8 x_{32}+7.7 x_{33}-d_{1}^{+}+d_{1}^{-}=y_{1} \\
170 \leq y_{1} \leq 220 \\
Z^{2}=50 x_{11}+65 x_{12}+62 x_{13}+60 x_{21}+55 x_{22}+58 x_{23} \\
+65 x_{31}+60 x_{32}+58 x_{33}-d_{2}^{+}+d_{2}^{-}=y_{2} \\
1550 \leq y_{2} \leq 1800 \\
\\
Z^{3}=10 x_{11}+8 x_{12}+9 x_{13}+8.5 x_{21}+9.5 x_{22}+8.5 x_{23} \\
+9.5 x_{31}+8.8 x_{32}+9 x_{33}-d_{3}^{+}+d_{3}^{-}=y_{3} \\
200 \leq y_{3} \leq 290 \\
d_{t}^{+}, d_{t}^{-} \geq 0, \quad t=1,2,3 \\
\text { and }(31) \text { to }(37)
\end{array}
$$


Again, considering the same weights $w_{t}$ as used in Model 3A for all $t=1,2,3$ and setting $\alpha_{t}=w_{t}$ for $t=1,2,3$ for deviation of goals and using Model 1B, Model 3 reduces to the following model (see Model 3B) as

Model 3B

$$
\begin{array}{r}
\min \frac{1}{50}\left(d_{1}^{+}+d_{1}^{-}\right)+\frac{1}{250}\left(d_{2}^{+}+d_{2}^{-}\right)+\frac{1}{90}\left(d_{3}^{+}+d_{3}^{-}\right) \\
+\frac{1}{50}\left(e_{1}^{+}+e_{1}^{-}\right)+\frac{1}{250}\left(e_{2}^{+}+e_{2}^{-}\right)+\frac{1}{90}\left(e_{3}^{+}+e_{3}^{-}\right) \\
\text {subject to } 7 x_{11}+8 x_{12}+7.5 x_{13}+8 x_{21}+7.2 x_{22}+8.4 x_{23} \\
+9 x_{31}+8 x_{32}+7.7 x_{33}-d_{1}^{+}+d_{1}^{-}=y_{1} \\
y_{1}-e_{1}^{+}+e_{1}^{-}=220 \\
170 \leq y_{1} \leq 220 \\
50 x_{11}+65 x_{12}+62 x_{13}+60 x_{21}+55 x_{22}+58 x_{23} \\
+65 x_{31}+60 x_{32}+58 x_{33}-d_{2}^{+}+d_{2}^{-}=y_{2} \\
y_{2}-e_{2}^{+}+e_{2}^{-}=1550 \\
1550 \leq y_{2} \leq 1800 \\
10 x_{11}+8 x_{12}+9 x_{13}+8.5 x_{21}+9.5 x_{22}+8.5 x_{23} \\
+9.5 x_{31}+8.8 x_{32}+9 x_{33}-d_{3}^{+}+d_{3}^{-}=y_{3} \\
y_{3}-e_{3}^{+}+e_{3}^{-}=290 \\
200 \leq y_{3} \leq 290 \\
d_{t}^{+}, d_{t}^{-}, e_{t}^{+}, e_{t}^{-} \geq 0, t=1,2,3 \\
\text { and }(31) \text { to }(37)
\end{array}
$$

Using the concept of utility function described in section "Transformation technique for multi-choice parameters like cost, supply, and demand to the equivalent form", Model 3 can be reformulated as follows.

The consideration of utility function depends on the DM. Here, we assume that goals 1, 2, and 3 follow the utility functions LLUF (Figure 1), RLUF (Figure 2), and S-shaped utility function as given in Figure 4, respectively. In the given example, the upper bound of variations $d_{1}^{+}, d_{1}^{-}, d_{2}^{+}, d_{2}^{-}, d_{3}^{+}, d_{3}^{-}$are 50,50, 250, 250, 90, 90, respectively, and the upper bounds of $f_{1}^{-}, f_{2}^{-}, f_{3}^{-}$are 1 . We find the weights as described in Section "Mathematical model" as follows: $w_{1}=\frac{1}{50}, w_{2}=\frac{1}{250}, w_{3}=\frac{1}{90}, \beta_{1}=\frac{50}{51}, \beta_{2}=\frac{250}{251}, \beta_{3}=\frac{90}{91}$.

With these supplied data, Model 3 can be reformulated as follows (see Model 3C):

Model 3C

$$
\begin{aligned}
& \min w_{1}\left(d_{1}^{+}+d_{1}^{-}\right)+\beta_{1} f_{1}^{-}+w_{2}\left(d_{2}^{+}+d_{2}^{-}\right)+\beta_{2} f_{2}^{-}+w_{3}\left(d_{31}^{+}+d_{32}^{+}+d_{32}^{+}\right)+\beta_{3} f_{3}^{-} \\
& \text {subject to } 7 x_{11}+8 x_{12}+7.5 x_{13}+8 x_{21}+7.2 x_{22}+8.4 x_{23} \\
& +9 x_{31}+8 x_{32}+7.7 x_{33}-d_{1}^{+}+d_{1}^{-}=y_{1} \\
& u_{1} \leq \frac{220-y_{1}}{50} \\
& f_{1}^{-}+u_{1}=1 \\
& 170 \leq y_{1} \leq 220
\end{aligned}
$$




$$
\begin{array}{r}
50 x_{11}+65 x_{12}+62 x_{13}+60 x_{21}+55 x_{22}+58 x_{23} \\
+65 x_{31}+60 x_{32}+58 x_{33}-d_{2}^{+}+d_{2}^{-}=y_{2} \\
u_{2} \leq \frac{y_{2}-1550}{250} \\
f_{2}^{-}+u_{2}=1 \\
1550 \leq y_{2} \leq 1800 \\
10 x_{11}+8 x_{12}+9 x_{13}+8.5 x_{21}+9.5 x_{22}+8.5 x_{23} \\
+9.5 x_{31}+8.8 x_{32}+9 x_{33}-d_{3}^{+}+d_{3}^{-}=y_{3} \\
u_{3}=(.4-0)\left(d_{31}-d_{32}\right) / 30+(1-.4)\left(d_{32}-d_{33}\right) / 40+(.85-1) d_{33} / 20 \\
y_{3}-d_{31}+d n_{31}=230, y_{3}-d_{32}+d n_{32}=270, y_{3}-d_{33}+d n_{33}=290 \\
d_{31} d n_{31}=0, d_{32} d n_{32}=0, d_{33} d n_{33}=0, \quad f_{3}^{-}+u_{3}=1 \\
u_{t} \geq 0, f_{t}^{-} \geq 0 \quad \forall t=1,2,3 \\
\text { and (31) to }(37)
\end{array}
$$

\section{Results and discussion for problem given in case 1}

Using LINGO software, we solved Models 3A, 3B, and 3C and reported the solution as follows:

The optimal solution of Model 3A is reported as

$x_{11}=0, x_{12}=9, x_{13}=1, x_{21}=0, x_{22}=0, x_{23}=9, x_{31}=6, x_{32}=0, x_{33}=0$; $Z^{1}=209.1, Z^{2}=1559, Z^{3}=214.5$.

The optimal solution of Model 3B is as follows:

$x_{11}=10, x_{12}=0, x_{13}=0, x_{21}=0, x_{22}=9, x_{23}=0, x_{31}=0, x_{32}=0, x_{33}=11$; $Z^{1}=219.50, Z^{2}=1633, Z^{3}=284.5$.

The optimal solution of Model $3 \mathrm{C}$ is also as follows:

$x_{11}=10, x_{12}=0, x_{13}=0, x_{21}=0, x_{22}=5, x_{23}=4, x_{31}=0, x_{32}=3, x_{33}=7$; $Z^{1}=217.5, Z^{2}=1593, Z^{3}=270.9$.

Here, the solution obtained in Model 3B is better compared with the solution of Model $3 \mathrm{~A}$, but the DM is not satisfied because in the proposed problem, satisfying the goal is not only the important notion but is also a utility factor to the DM which is important for the decision-making (management) problem. When the utility value is more important rather than the benefit, then the solutions obtained in Model 3A or in Model 3B are not satisfactory to the DM to make an appropriate decision. The marketing survey indicates that the higher utility value of goal 3 will increase the number of customers to the network service provider company. The solution obtained in Model 3C demonstrated the high utility value of goal 3 , whenever the other two models failed to give satisfactory results (Table 1). In this context, we may suggest that the utility function approach provided better result compared with other results obtained in classical techniques like GP and RMCGP. 
Table 1 Comparison of achieved goals obtained from the different methods

\begin{tabular}{lccc}
\hline Method & $\begin{array}{c}\text { Achievement of } \\
\text { goal 1 (\%) }\end{array}$ & $\begin{array}{c}\text { Achievement of } \\
\text { goal 2 (\%) }\end{array}$ & $\begin{array}{c}\text { Achievement of } \\
\text { goal 3 (\%) }\end{array}$ \\
\hline GP & 75 & 95 & 40 \\
RMCGP & 99 & 60 & 90 \\
Utility approach & 98 & 80 & 99 \\
\hline
\end{tabular}

Case 2

Let us consider the following MCMTP (see Model 3) with two objectives:

\section{Model 4}

Goal 1: $z_{1}=\{5$ or 7$\} x_{11}+8 x_{12}+\{7$ or 6 or 10$\} x_{13}+\{6$ or 8$\} x_{21}+8 x_{22}+10 x_{23}$ with goal as $[150,200]$, more is better, but follows S-shape utility function (Figure 5).

Goal $2: z_{2}=15 x_{11}+\{18$ or 16$\} x_{12}+17 x_{13}+16 x_{21}+\{18$ or 20$\} x_{22}+20 x_{23}$

with goal as $[400,500]$, less is better, follows LLUF:

subject to $x_{11}+x_{12}+x_{13} \leq\{11$ or 13 or 12 or 16$\}$

$$
\begin{array}{r}
x_{21}+x_{22}+x_{23} \leq\{14 \text { or } 13\} \\
x_{11}+x_{21} \geq\{8 \text { or } 7\} \\
x_{12}+x_{22} \geq\{7 \text { or } 8 \text { or } 6\} \\
x_{13}+x_{23} \geq 9 \\
x_{i j} \geq 0 \forall i=1,2 \text { and } j=1,2,3 .
\end{array}
$$

Model 4 is equivalent to the following model (see Model 5).

\section{Model 5}

Goal 1: $Z_{1}=\left\{5 z_{11}^{11}+7\left(1-z_{11}^{11}\right)\right\} x_{11}+8 x_{12}+\left\{7 z_{11}^{12} z_{11}^{13}+6 z_{11}^{12}\left(1-z_{11}^{13}\right)\right.$

$$
\left.+10 z_{11}^{13}\left(1-z_{11}^{12}\right)\right\} x_{13}+\left\{6 z_{11}^{21}+8\left(1-z_{11}^{21}\right)\right\} x_{21}+8 x_{22}+10 x_{23}
$$

with goal as $[150,200]$, more is better, but follows S-shape utility function (Figure 5).

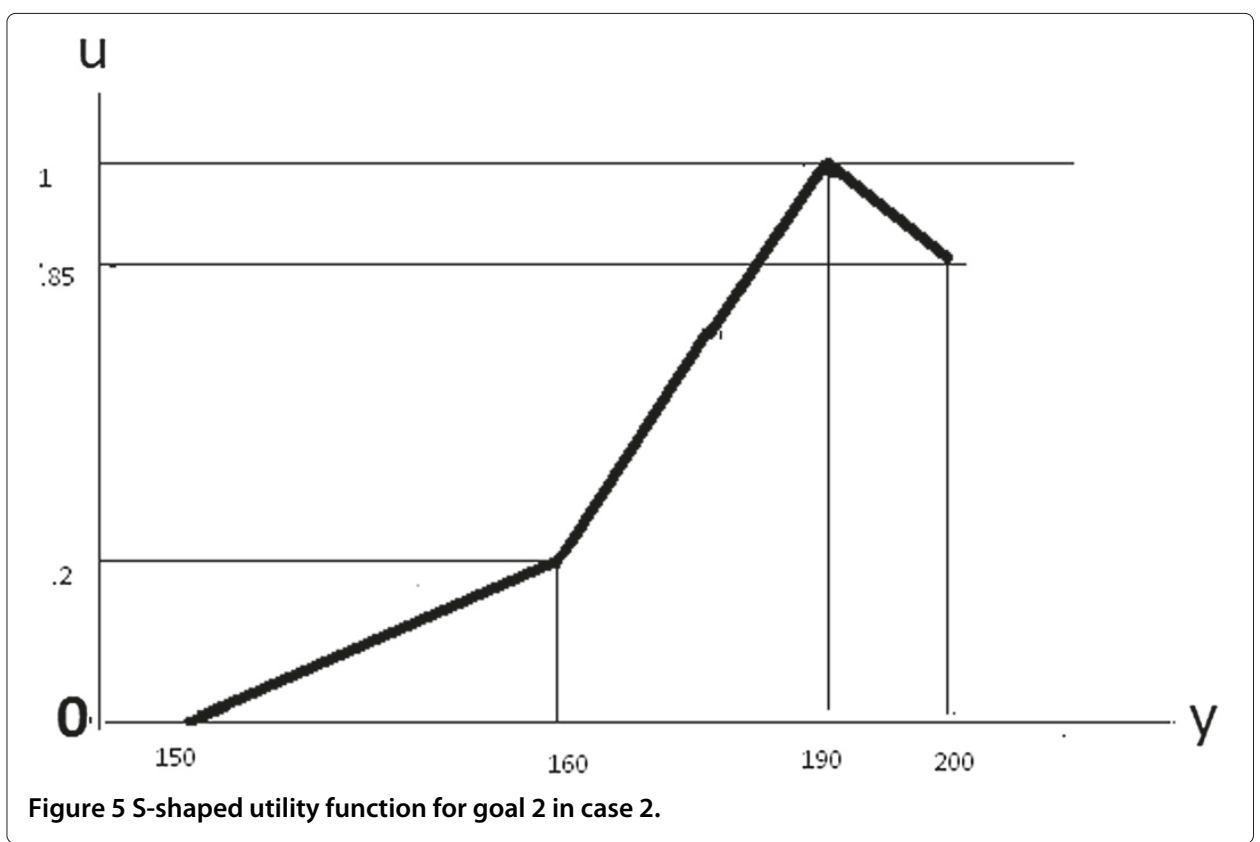


Goal $2: Z_{2}=15 x_{11}+\left\{18 z_{12}^{11}+16\left(1-z_{12}^{11}\right)\right\} x_{12}+17 x_{13}+16 x_{21}$

$$
+\left\{18 z_{12}^{12}+20\left(1-z_{12}^{12}\right)\right\} x_{22}+20 x_{23}
$$

with goal as $[400,500]$, less is better, follows LLUF:

$$
\begin{array}{r}
\text { subject to } x_{11}+x_{12}+x_{13} \\
\leq\left\{1 z_{1}^{11} z_{1}^{12}+13 z_{1}^{11}\left(1-z_{1}^{12}\right)+12 z_{1}^{12}\left(1-z_{1}^{11}\right)+16\left(1-z_{1}^{11}\right)\left(1-z_{1}^{12}\right)\right\} \\
x_{21}+x_{22}+x_{23} \leq\left\{14 z_{2}^{11}+16\left(1-z_{2}^{11}\right)\right\} \\
x_{11}+x_{21} \geq\left\{8 z^{11}+7\left(1-z^{11}\right)\right\} \\
x_{12}+x_{22} \geq\left\{7 z^{21} z^{22}+8 z^{21}\left(1-z^{22}\right)+6 z^{22}\left(1-z^{21}\right)\right\} \\
x_{13}+x_{23} \geq 9 \\
x_{i j} \geq 0 \forall i=1,2 \text { and } j=1,2,3 . \\
z_{11}^{12}+z_{11}^{13} \geq 1 \\
z^{21}+z^{22} \geq 1 \\
z_{11}^{11}, z_{11}^{12}, z_{11}^{13}, z_{12}^{11}, z_{12}^{12}=0 \text { or } 1 \\
z_{1}^{11}, z_{1}^{12}, z_{2}^{11}=0 \text { or } 1 \\
z^{11}, z^{21}, z^{22}=0 \text { or } 1
\end{array}
$$

In the given problem in Model 5, the deviations of goal 1, goal 2 are 50, 100 respectively. By considering the weights $w_{1}=\frac{1}{50}, w_{2}=\frac{1}{100}$ for the Model 1A, Model 5 reduces to Model $5 A$ as

Model 5 A

$$
\begin{array}{r}
\min \frac{1}{50}\left(d_{1}^{+}+d_{1}^{-}\right)+\frac{1}{100}\left(d_{2}^{+}+d_{2}^{-}\right) \\
\text {subject to }\left\{5 z_{11}^{11}+7\left(1-z_{11}^{11}\right)\right\} x_{11}+8 x_{12}+\left\{7 z_{11}^{12} z_{11}^{13}+6 z_{11}^{12}\left(1-z_{11}^{13}\right)\right. \\
\left.+10 z_{11}^{13}\left(1-z_{11}^{12}\right)\right\} x_{13}+\left\{6 z_{11}^{21}+8\left(1-z_{11}^{21}\right)\right\} x_{21}+8 x_{22}+10 x_{23}-d_{1}^{+}+d_{1}^{-}=y_{1}, \\
150 \leq y_{1} \leq 200 \\
15 x_{11}+\left\{18 z_{12}^{11}+16\left(1-z_{12}^{11}\right)\right\} x_{12}+17 x_{13}+16 x_{21} \\
+\left\{18 z_{12}^{12}+20\left(1-z_{12}^{12}\right)\right\} x_{22}+20 x_{23}-d_{2}^{+}+d_{2}^{-}=y_{2}, \\
400 \leq y_{2} \leq 500 \\
d_{t}^{+}, d_{t}^{-} \geq 0, t=1,2 \\
\text { and }(44) \text { to }(54)
\end{array}
$$

Again, considering the same weights $w_{t}$ as used in Model 3A for all $t=1,2$ and the weights $\alpha_{t}=w_{t}$ for $t=1,2$ for deviation of goals and using the Model 1B, Model 5 reduces to Model $5 B$ as 
Model Ex. 5B

$$
\begin{array}{r}
\min \frac{1}{50}\left(d_{1}^{+}+d_{1}^{-}\right)+\frac{1}{100}\left(d_{2}^{+}+d_{2}^{-}\right)+ \\
+\frac{1}{50}\left(e_{1}^{+}+e_{1}^{-}\right)+\frac{1}{100}\left(e_{2}^{+}+e_{2}^{-}\right) \\
\text {subject to }\left\{5 z_{11}^{11}+7\left(1-z_{11}^{11}\right)\right\} x_{11}+8 x_{12}+\left\{7 z_{11}^{12} z_{11}^{13}+6 z_{11}^{12}\left(1-z_{11}^{13}\right)\right. \\
\left.+10 z_{11}^{13}\left(1-z_{11}^{12}\right)\right\} x_{13}+\left\{6 z_{11}^{21}+8\left(1-z_{11}^{21}\right)\right\} x_{21}+8 x_{22}+10 x_{23}-d_{1}^{+}+d_{1}^{-}=y_{1}, \\
y_{1}-e_{1}^{+}+e_{1}^{-}=200, \\
150 \leq y_{1} \leq 200, \\
15 x_{11}+\left\{18 z_{12}^{11}+16\left(1-z_{12}^{11}\right)\right\} x_{12}+17 x_{13}+16 x_{21} \\
+\left\{18 z_{12}^{12}+20\left(1-z_{12}^{12}\right)\right\} x_{22}+20 x_{23}-d_{2}^{+}+d_{2}^{-}=y_{2}, \\
y_{2}-e_{2}^{+}+e_{2}^{-}=400, \\
400 \leq y_{2} \leq 500, \\
d_{t}^{+}, d_{t}^{-}, e_{t}^{+}, e_{t}^{-} \geq 0, t=1,2 \\
\text { and }(44) \text { to }(54)
\end{array}
$$

Let us solve the proposed problem (see Model 3) using the concept of utility function. The consideration of utility function depends on the DM. Here we assume that goal 1 and goal 2 follow the S-shaped utility function given in Figure 5 and the utility functions LLUF (Figure 1), respectively. In the given example, the upper bound of variations $d_{1}^{+}, d_{1}^{-} ; d_{2}^{+}, d_{2}^{-}$are 50, 100 respectively, and the upper bounds of $f_{1}^{-}, f_{2}^{-}$are 1 . We find the weights as suggested in Section "Mathematical model" as follows: $w_{1}=\frac{1}{50}, \beta_{1}=\frac{50}{51}$, $w_{2}=\frac{1}{100}, \beta_{2}=\frac{100}{101}$.

With these supplied data, Model 5 can be formulated as follows:

Model 5C

$$
\begin{array}{r}
\min w_{1}\left(d_{11}^{+}+d_{12}^{+}+d_{13}^{+}\right)+\beta_{1} f_{1}^{-}+w_{2}\left(d_{2}^{+}+d_{2}^{-}\right)+\beta_{2} f_{2}^{-} \\
\text {subject to }\left\{5 z_{11}^{11}+7\left(1-z_{11}^{11}\right)\right\} x_{11}+8 x_{12}+\left\{7 z_{11}^{12} z_{11}^{13}+6 z_{11}^{12}\left(1-z_{11}^{13}\right)\right. \\
\left.+10 z_{11}^{13}\left(1-z_{11}^{12}\right)\right\} x_{13}+\left\{6 z_{11}^{21}+8\left(1-z_{11}^{21}\right)\right\} x_{21}+8 x_{22}+10 x_{23}-d_{1}^{+}+d_{1}^{-}=y_{1}, \\
u_{1}=(.2-0)\left(d_{11}-d_{12}\right) / 10+(1-.2)\left(d_{12}-d_{13}\right) / 30+(.85-1) d_{13} / 10 \\
f_{3}-d_{11}+d n_{11}=160, f_{3}-d_{12}+d n_{12}=190, f_{3}-d_{13}+d n_{13}=200 \\
d_{11} d n_{11}=0, d_{12} d n_{12}=0, d_{13} d n_{13}=0, \\
f_{1}+u_{1}=1, \\
15 x_{11}+\left\{18 z_{12}^{11}+16\left(1-z_{12}^{11}\right)\right\} x_{12}+17 x_{13}+16 x_{21} \\
+\left\{18 z_{12}^{12}+20\left(1-z_{12}^{12}\right)\right\} x_{22}+20 x_{23}-d_{2}^{+}+d_{2}^{-}=y_{2}, \\
u_{2} \leq \frac{200-y_{2}}{100}, \\
f_{2}+u_{2}=1, \\
400 \leq y_{2} \leq 500, \\
u_{t} \geq 0, f_{t} \geq 0 \quad \forall t=1,2 \\
\text { and }(44) \text { to }(54)
\end{array}
$$




\section{Results and discussion for problem given in case 2}

Solving the model presented in Model 5A, the optimal solution of the Model 5A is reported as $x_{11}=7, x_{12}=5, x_{13}=0, x_{21}=0, x_{22}=1, x_{23}=10$, and the values of the objective functions are $Z^{1}=197, Z^{2}=405$.

The selection of the choices corresponding to the optimal solution is as follows:

$c_{11}^{1}=7, c_{12}^{1}=8, c_{13}^{1}=10, c_{21}^{1}=8, c_{22}^{1}=8, c_{23}^{1}=10$

$c_{11}^{2}=15, c_{12}^{2}=16, c_{13}^{2}=17, c_{21}^{2}=16, c_{22}^{2}=18, c_{23}^{2}=20$

$a_{1}=16, a_{2}=14, b_{1}=7, b_{2}=6, b_{3}=9$

Solving the model presented in Model 5B, we have listed the following solution:

$x_{11}=7, x_{12}=6, x_{13}=0, x_{21}=0, x_{22}=0, x_{23}=10$, and the values of the objective functions are $Z^{1}=197, Z^{2}=401$.

The selection of the choices corresponding to the optimal solution is as follows:

$c_{11}^{1}=7, c_{12}^{1}=8, c_{13}^{1}=10, c_{21}^{1}=6, c_{22}^{1}=8, c_{23}^{1}=10$

$c_{11}^{2}=15, c_{12}^{2}=16, c_{13}^{2}=17, c_{21}^{2}=16, c_{22}^{2}=18, c_{23}^{2}=20$

$a_{1}=16, a_{2}=14, b_{1}=7, b_{2}=8, b_{3}=9$

Solving the model presented in Model 5C, we obtained the solution listed below:

$x_{11}=6, x_{12}=3, x_{13}=0, x_{21}=0, x_{22}=3, x_{23}=10$, and the values of the objective functions are $Z^{1}=190, Z^{2}=404$.

The selection of the choices corresponding the optimal solution is as follows:

$c_{11}^{1}=7, c_{12}^{1}=8, c_{13}^{1}=10, c_{21}^{1}=6, c_{22}^{1}=8, c_{23}^{1}=10$

$c_{11}^{2}=15, c_{12}^{2}=18, c_{13}^{2}=17, c_{21}^{2}=16, c_{22}^{2}=20, c_{23}^{2}=20$

$a_{1}=12, a_{2}=14, b_{1}=7, b_{2}=6, b_{3}=9$

Table 2 helps us to conclude that the solution of the MCMTP obtained in Model 5B is better compared with the solution of Model 5A, but the DM is not satisfied because in the proposed problem satisfying the goal is not only the important notion, but is also a utility factor to the DM which is important for the decision-making (management) problem. When the utility value is more important rather than the benefit, then the solutions obtained in Model 5A or in Model 5B are not satisfactory to the DM to make the appropriate decision. The solution obtained in the Model 5C demonstrated the high utility value of goal 2, whenever the other two models failed to give satisfactory results. In this context, we may suggest that the utility function approach provided better result compared with other results.

\section{Conclusions}

In this paper, we have considered MCMTP where the cost, demand, and supply coefficients are multi-choice type. Another important notion of this study is to give an impression of goal preferences of the DM. The approach of utility function is the most useful skill for representing the DM's preferences. The concept of utility in this paper

Table 2 Comparison of achieved goals obtained from different methods

\begin{tabular}{lcc}
\hline Method & $\begin{array}{c}\text { Achievement of } \\
\text { goal 1 (\%) }\end{array}$ & $\begin{array}{c}\text { Achievement of } \\
\text { goal 2 (\%) }\end{array}$ \\
\hline GP & 85 & 96 \\
RMCGP & 85 & 98 \\
Utility approach & 100 & 95 \\
\hline
\end{tabular}


proposes a new approach for extending the utilization of real-life MCMTP. The MCMTP gives a new direction to handle the real-life transportation problems when the transportation parameters are multi-choice in nature. The numerical examples presented in this paper explored the applicability and suitability for solving MOTP and MCMTP and also for representing the DM's preferences. In addition, the proposed method can be used as a decision-making aid for multi-choice multi-objective decision-making problem that occurred in the real-life purposes, like economical, agricultural, industrial management, and military. In particular, in case of incomplete information, the DM can use the proposed method to set the goals according to their own utility functions, while the proposed method can easily find the better solution than the previous methods (GP, RMCGP) used to solve MCMTP which is shown by the proposed examples in this paper.

Received: 31 December 2013 Accepted: 6 April 2014

Published: 30 April 2014

References

1. Chang, C-T: Multi-choice goal programming. Omega. 35, 389-396 (2007)

2. Chang, C-T: Revised multi-choice goal programming. Appl. Math. Model. 32, 2587-2595 (2008)

3. Mahapatra, DR, Roy, SK, Biswal, MP: Multi-choice stochastic transportation problem involving extreme value distribution. Appl. Math. Modell. 37, 2230-2240 (2013)

4. Roy, SK, Mahapatra, DR, Biswal, MP: Multi-choice stochastic transportation problem with exponential distribution. J. Uncert. Syst. 6(3), 200-213 (2013)

5. Al-nowaihi, A, Bradley, I, Dhami, S: The utility function under prospect theory. Econ. Lett. 99, 337-339 (2008)

6. Yu, BW-T, Pang, WK, Troutt, MD, Hou, SH: Objective comparisons of the optimal portfolios corresponding to different utility functions. Eur. J. Oper. Res. 199, 604-610 (2009)

7. Podinovski, W: Set choice problems with incomplete information about the preferences of the decision maker. Eur. J. Oper. Res. 207, 371-379 (2010)

8. Charnes, A, Cooper, WW, Ferguson, RO: Optimal estimation of executive compensation by linear programming. Manage. Sci. 1, 138-151 (1955)

9. Charnes, A, Cooper, WW: Management Model and Industrial Application of Linear Programming, Vol. 1. Wiley, New York (1961)

10. Lee, SM: Goal Programming for Decision Analysis. Auerbach, Philadelphia (1972)

11. Ignizio, JP: Introduction to Linear Goal Programming. Sage, Beverly Hills (1985)

12. Tamiz, M, Jones, D, Romero, C: Goal programming for decision making: an overview of the current state-of-the-art. Eur. J. Oper. Res. 111, 567-581 (1998)

13. Romero, C: Extended lexicographic goal programming: a unifying approach. Omega. 29, 63-71 (2001)

14. Lai, Y-J, Hwang, C-L: Fuzzy Multiple Objective Decision Making: Methods and Applications. Springer, Berlin (1994)

15. Chang, C-T: An approximation approach for representing S-shaped membership functions. IEEE Trans. Fuzzy Syst. 18, 412-424 (2010)

16. Kettani, O, Aouni, B, Martel, JM: The double role of the weight factor in the goal programming model. Comput. Oper. Res. 31, 1833-1845 (2004)

17. Chang, C-T: Mixed binary interval goal programming. J. Oper. Res. Soc. 57, 469-473 (2006)

doi:10.1186/2195-5468-2-11

Cite this article as: Maity and Roy: Solving multi-choice multi-objective transportation problem: a utility function approach. Journal of Uncertainty Analysis and Applications 2014 2:11.

\section{Submit your manuscript to a SpringerOpen ${ }^{\circ}$ journal and benefit from:}

- Convenient online submission

- Rigorous peer review

- Immediate publication on acceptance

- Open access: articles freely available online

- High visibility within the field

- Retaining the copyright to your article

Submit your next manuscript at $>$ springeropen.com 